\title{
REZENSIONEN
}

\section{Die Fallstricke des deutschen Wahlsystems: gut analysiert}

\author{
Behnke, Joachim: Das Wahlsystem der Bundesrepublik Deutschland. Logik, Technik und Praxis \\ der Verhältniswahl, Nomos Verlagsgesellschaft, Baden-Baden 2007, 249 Seiten, € 34,-.
}

Im Juni 2009 erregte eine Simulationsstudie von Joachim Behnke großes Aufsehen. ${ }^{1}$ Der Autor, Professor für Politikwissenschaft an der Zeppelin-University in Friedrichshafen und Sprecher des Arbeitskreises „Empirische Methoden der Politikwissenschaft“ der DVPW, kam zu dem Ergebnis, die Union werde wegen des vermutlich großen Abstandes zur SPD viele Überhangmandate erringen. Daher plädierte Behnke für eine Änderung des Wahlsystems vor der Bundestagswahl - leider vergebens. Der Gesetzentwurf der Bundestagsfraktion von Bündnis 90 / Die Grünen, der Überhangmandate ausschließen wollte (und zwar dadurch, dass die Direktmandate von allen Mandaten, die einer Partei zustehen, abgezogen werden, ehe die Verteilung auf die Länder erfolgt), bekam keine Mehrheit. Die SPD votierte mehrheitlich gegen ihn, um nicht den Bruch des Regierungsbündnisses zu riskieren.

Behnke wusste, wovon er sprach. In seiner hier anzuzeigenden preisgekrönten Bamberger Habilitationsschrift spielt das komplizierte System der Überhangmandate und die - berechtigte - Kritik daran eine beträchtliche Rolle. Wer das Buch wegen des eingängigen Titels erwirbt, sitzt einem Etikettenschwindel auf: Es handelt sich um eine gelehrte Spezialstudie. Im ersten Teil geht es um normative und pragmatische Begründungen für ein spezifisches Wahlsystem, im zweiten um die Technik des Verrechnungsverfahrens, im dritten um die Praxis der Verhältniswahl am Beispiel der Bundesrepublik Deutschland. Das ist insofern nicht ganz konsequent, als das konkrete Verrechnungsverfahren ein Teil der deutschen Praxis ist (und mehrfach geändert wurde: 1985 von d'Hondt zu Hare / Niemeyer und 2008 von Hare / Niemeyer zu Sainte-Laguë / Schepers). Das deutsche (vor einigen Jahren auch in Neuseeland eingeführte) Wahlsystem erfreut sich im Ausland zum Teil großer Beliebtheit, sei es, weil das Referenzmodell funktioniert, sei es, weil manche (irrigerweise) denken, das Verfahren stelle einen annehmbaren Kompromiss zwischen Mehrheits- und Verhältniswahl dar. Der Autor hält es zu Recht für sinnvoll, das Wahlsystem mit seinen Fallstricken beständig auf seine Legitimation hin zu überprüfen, auch wenn es keine größere Kritik auslöst. Behnkes Stärke besteht in der Verknüpfung von Wahlsystematik und Wahlsoziologie.

Wer seinen Argumentationsgang nachvollziehen will, muss mehr als mathematische Grundlagenkenntnisse besitzen. Das gilt weniger für den ersten Teil, in dem der Autor unter anderem über die Vor- und Nachteile der Wahlverfahren reflektiert. Dabei unterscheidet Behnke zwischen einem „taktischen“ und einem „strategischen Wählen“. In beiden Fällen weicht der Wähler von seiner generellen Parteipräferenz ab. Beim taktischen Wählen wird das Votum anderer Stimmbürger nicht berücksichtigt, im Gegensatz zum strategischen Wählen. Behnke gelangt zu folgendem Ergebnis: „Unter dem Aspekt, die Wirkungen takti-

1 Siehe auch seinen Beitrag in diesem Heft der ZParl. 
schen [und strategischen] Wahlverhaltens so gering wie möglich zu halten, ist daher ein Verhältniswahlsystem einem Mehrheitswahlsystem auf jeden Fall vorzuziehen“ (S. 97). Er muss jedoch selber einräumen, dass unter den Bedingungen der Verhältniswahl, etwa bei der Existenz der Fünfprozenthürde, ein Teil der Wähler abgeschreckt wird, für eine kleine Partei zu votieren („Fallbeil“-Effekt), ein anderer Teil sich dazu jedoch gerade ermuntert fühlt, damit sie in das Parlament einzieht („Leih“- oder „Stützstimme“) und mit seiner „eigentlichen" Partei eine Koalition eingehen kann. Anreize zu strategischem Wählerverhalten bestehen demnach auch bei einem Verhältniswahlsystem.

Der zweite Teil über das angemessene Verrechnungsverfahren beim Proportionalwahlverfahren ist technizistischer Natur, der Unterschied bei der Umrechnung von Stimmen in Mandate nur minimaler Natur. ${ }^{2}$ Generell wird zwischen den Divisorverfahren (zum Beispiel dem Höchstzahlverfahren nach d'Hondt) und den Quota-Verfahren unterschieden. Mit Quota ist die Anzahl der Stimmen gemeint, die für ein Mandat genügen. Behnke will das Verfahren mit der größten Proportionalität ermitteln. Der mathematische Aufwand, den er betreibt, steht in keinem angemessenen Verhältnis zum Ertrag. Sein Ergebnis, das auf höchster Präzision basiert: „Wenn man z.B. den Wahlrechtsgrundsatz der Gleichheit auf einen möglichst gleichen Erfolgswert für alle Parteien bezieht, dann müsste, wenn man das Prinzip sehr streng auslegt, entweder das Verfahren von Sainte-Laguë oder das von Hill statt des Hare / Niemeyer-Verfahrens gewählt werden, je nachdem, ob man den absoluten oder den relativen Unterschied zu minimieren versucht" (S. 176). So ist es bekanntlich geschehen. Dabei wurde aber vergessen: Für die Legitimität des jeweiligen Verfahrens spielt auch die Verständlichkeit und Nachvollziehbarkeit eine tragende Rolle. Sie ist bei Hare / Niemeyer gegeben, nicht jedoch bei Sainte-Laguë/ Schepers.

Der dritte Teil ist der aufschlussreichste. Im Vordergrund stehen zwei Besonderheiten des deutschen Wahlsystems: die Überhangmandatsregelung und die Zweistimmenkonstruktion. Beide Spezifika sind höchst kritikbedürftig. Der Autor zeichnet souverän und differenziert die Ursachen für das Entstehen der Überhangmandate nach. Sie gehen nur in wenigen Fällen auf das unterschiedliche Erst- und Zweitstimmenvotum zurück, sondern vielmehr auf die Existenz anderer Faktoren, die häufig zusammenwirken (vor allem: starke Drittpartei; überproportional viele Wahlkreise in einem Bundesland; niedrige Wahlbeteiligung). Das starke Auftreten von Überhangmandaten in den neuen Bundesländern (46 von 56) verwundert daher nicht. Behnke erhellt auch das Paradoxon des inversen Erfolgswertes: Mehr Stimmen können zu weniger Mandaten (und weniger Stimmen zu mehr Mandaten) führen. Der Autor erörtert verschiedene Möglichkeiten zur Beseitigung, zur massiven Verringerung oder zur Kompensation der Überhangmandate (unter anderem Abzug „überschüssiger" Direktmandate, Senkung des Anteils der Direktmandate, Verrechnung von Überhangmandaten mit Listenmandaten derselben Partei in anderen Bundesländern, Einführung von Ausgleichsmandaten wie in den Bundesländern). Sein Reformvorschlag - die Einführung von Mehrpersonenwahlkreisen - ist ausgesprochen kompliziert und wenig praktikabel.

2 Allerdings sieht dies mit Blick auf Überhangmandate anders aus. Nach dem Verfahren d'Hondt hätte die SPD bei der Bundestagswahl 2005 elf Mandate erhalten, die Union lediglich sechs. Damit wäre die SPD auf den gleichen Mandatsanteil gekommen wie die Union. 
Die Zweistimmenkonstruktion wird unter dem Gesichtspunkt erörtert, welche taktischen und strategischen Möglichkeiten ein Stimmensplitting bietet. Leider reizt der Autor die Thematik dabei nicht voll aus. Einen Reformvorschlag unterbreitet Behnke beim Zweistimmensystem nicht, wohl deswegen, weil er keine großen Möglichkeiten zur Manipula-tion sieht. Die Wähler empfinden das jedoch vielfach anders und neigen zum Stimmensplitting auch deshalb, weil sie glauben, dadurch besonders geschickt zu handeln. Gegen eine Abschaffung des Zweistimmensystems, wie sie unter anderem der Rezensent fordert, sprächen "gewichtige demokratietheoretische Gründe“ (S. 202), die Behnke nicht eigens benennt. Der Ertrag der Ausführungen zum Zweistimmensystem fällt suboptimal aus.

Sollte die Union nur dank möglicher Überhangmandate gemeinsam mit den Liberalen bei der Bundestagswahl 2009 eine Mandatsmehrheit erlangen, könnte ein Legitimationsproblem eintreten. Behnke nimmt zu Recht kein Blatt vor den Mund, spricht gar, etwas überzogen, von einer „demokratietheoretischen Katastrophe“ (S. 235). „So bleibt also nur zu hoffen, dass sich die Einsicht, dass eine Reform des Wahlgesetzes nicht nur überfällig, sondern auch ein Gebot politischer und demokratischer Klugheit ist, in nächster Zeit durchsetzen wird“ (S. 235). Diese Hoffnung geht für die nächste Wahlperiode nach 2009 auf, denn das Bundesverfassungsgericht hat in seinem Urteil vom 3. Juli 2008 dem passiv gebliebenen Gesetzgeber zu Recht aufgetragen, das Wahlverfahren bis zum 30. Juni 2011 so zu ändern, dass ein ,inverser Erfolgswert“ unmöglich gemacht wird. Der Rezensent seinerseits hofft: Mögen Überhangmandate das Ergebnis nicht in das Gegenteil verkehren.

Eckhard Jesse

\section{Bundeswahl- und Parteiengesetz: instruktiv kommentiert}

Schreiber, Wolfgang: Bundeswahlgesetz (BWahlG). Kommentar, 8. Auflage, Carl Heymanns Verlag, Köln 2009, 1125 Seiten, € 148,-.

Ipsen, Jörn (Hrsg.): Gesetz über die politischen Parteien (ParteienG). Kommentar, Verlag C.H. Beck, München 2008, 588 Seiten, € 88,-.

Wahlrechtsfragen sind oft Machtfragen, jedoch nicht immer, wie das folgende Beispiel zeigt: Der Bundeswahlausschuss, dem der Bundeswahlleiter vorsteht, hat auch bei der diesjährigen Bundestagswahl einige Parteien aus formalen Gründen nicht zugelassen, so die Grauen, die anarchistische APPD und die Freie Union von Gabriele Pauli. Solche Fragen betreffen das Wahlgesetz ebenso wie das Parteiengesetz.

Im Jahr 1975 wurde für die Bundestagswahl 1976 zum ersten Mal das „Handbuch des Wahlrechts zum Deutschen Bundestag“ auf den Markt gebracht - unter der Ägide von Wolfgang Schreiber, des damaligen Wahlrechtsreferenten im Bundesministerium des Innern. Der Kommentar zum Bundeswahlrecht von Karl-Heinz Seifert wurde danach nicht mehr publiziert. ${ }^{1}$ Vor jeder folgenden Bundestagswahl - abgesehen von der vorgezogenen 2005

1 Vgl. Karl-Heinz Seifert, Bundeswahlrecht. Kommentar, 3. Auflage, München 1976. 